\title{
w-Hosoya polynomials for Pentagonal Chains
}

Ali Aziz Ali

\section{aliazizali1933@yahoo.com}

Academic

University of Mosul

Received on: : 17/04/2011

\section{Shwan Salou Abdul Ilyas}

Directorate of General Education

Nineveh

\section{Accepted on: 21/06/2011}

\begin{abstract}
Properties of the width distance in graphs are given in this paper. The w-Hosoya Polynomials of straight pentagonal chains and of alternate pentagonal chains are obtained with Wiener indices of the width distance of such graphs.

Keywords: Width distance, w-Hosoya polynomial, Pentagonal Chain.

متعددات حدود هوسويا-w لسلاسل حلقات خماسية

$$
\text { مديرية التربية العامة / مينة نينوى سلو للبنين عبدال إلياس }
$$

تاريخ قبول البحث: 2011/06/21

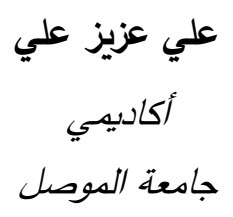

تاريخ استلام البحث: 2011/04/17

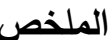

تضمن هذا البحث إعطاء العديد من خواص المسافة العرضية في البيانات، وشمل البحث على إيجاد متعددات حدود هوسويا-w لبيانات سلاسل خطية بحلقات خماسية وكذلك لسلاسل ذات حلقات خماسية متتاوبة، إضافة إلى الحصول على صيخ لاليل وينر نسبة إلى المسافة العرضية لهذه البيانات.

الكلمات المفتاحية : المسافة العرضية ، متعددة حدود هوسويا-W ، السلسلة الخماسية.

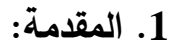

بخصوص ما ورد من مفاهيم في نظرية البيان نشير إلى المصادر [2, 5, 6]، وللاطلاع على المفاهيم

التي تخص متعددات حدود هوسويا ودليل وينر نشير إلى المصادر [7,8,9]. وللتعرف على المفاهيم التي تتعلق بالمسافة العرضية ومتعددات حدود هوسويا-W وبعض النتائج التي أوجدها الباحثون في هذا المجال، نثير إلى ولى

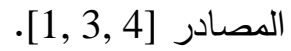

ليكن v،u رأسين مختلفين في بيان متصل G(u,v) على تعرف أنها مجموعة من الدروب الحاوية

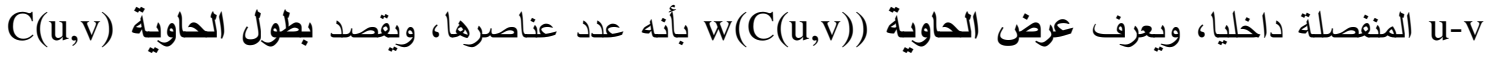
بأنه الأعظم لأطول الدروب u-v في الحاوية ويرمز له (C(u,v)
\end{abstract}




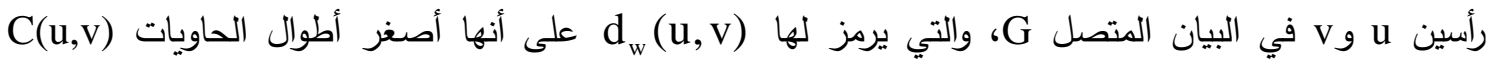

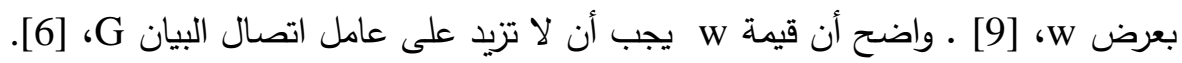
الاختلاف المركزي، والقطر ونصف القطر نسبة للمسافة العرضية تعرف بأسلوب مشابه لتعاريفها نسبة

للمسافة القياسية (الاعتيادية).

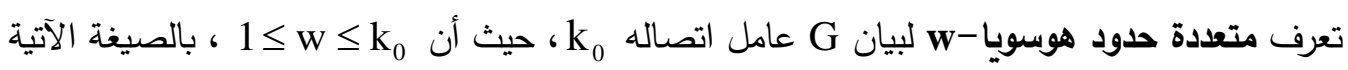
$\mathrm{H}_{\mathrm{w}}(\mathrm{G} ; \mathrm{x})=\sum_{\mathrm{u}, \mathrm{v} \in \mathrm{V}} \mathrm{x}^{\mathrm{d}_{\mathrm{w}}(\mathrm{u}, \mathrm{v})}$

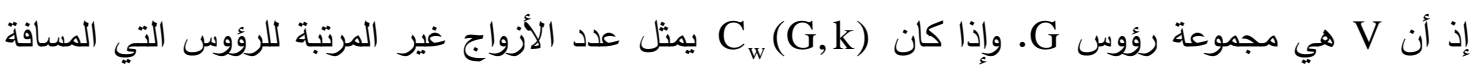

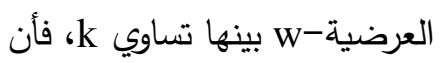

$$
\mathrm{H}_{\mathrm{w}}(\mathrm{G} ; \mathrm{x})=\sum_{\mathrm{k} \geq 1}^{\delta_{\mathrm{w}}} \mathrm{C}_{\mathrm{w}}(\mathrm{G}, \mathrm{k}) \mathrm{x}^{\mathrm{k}},
$$

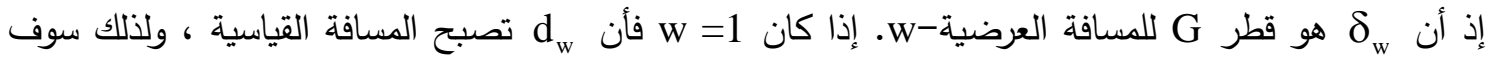

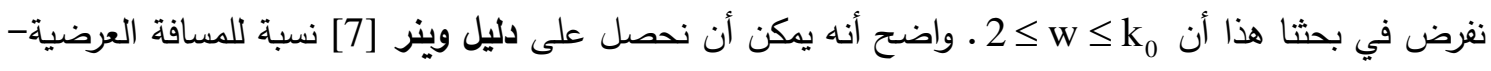

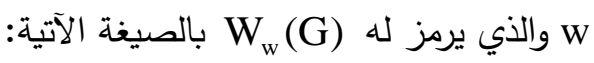

$$
\mathrm{W}_{\mathrm{w}}(\mathrm{G})=\left.\frac{\mathrm{d}}{\mathrm{dx}} \mathrm{H}_{\mathrm{w}}(\mathrm{G} ; \mathrm{x})\right|_{\mathrm{x}=1}=\sum_{\mathrm{k} \geq 2}^{\delta_{\mathrm{w}}} \mathrm{kC}_{\mathrm{w}}(\mathrm{G}, \mathrm{k})
$$

نعرف متعددة حدود هوسويا w للرأس vي G بالصيغة

$$
\mathrm{H}_{\mathrm{w}}(\mathrm{v}, \mathrm{G} ; \mathrm{x})=\sum_{\mathrm{k} \geq 2} \mathrm{C}_{\mathrm{w}}(\mathrm{v}, \mathrm{G}, \mathrm{k}) \mathrm{x}^{\mathrm{k}},
$$

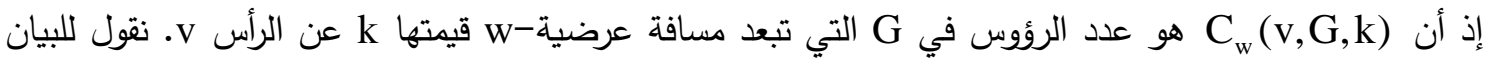

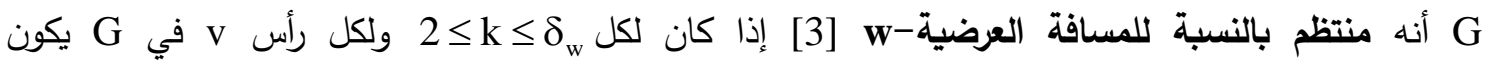

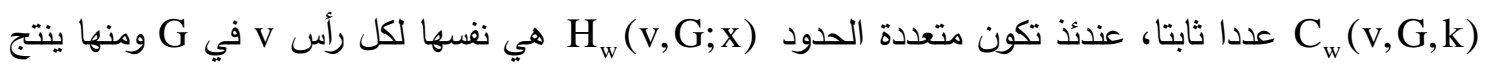

$$
\mathrm{H}_{\mathrm{w}}(\mathrm{G} ; \mathrm{x})=\frac{1}{2} \mathrm{pH}_{\mathrm{w}}(\mathrm{v}, \mathrm{G} ; \mathrm{x}), \mathrm{w} \geq 2,
$$$$
\text { لهذا النوع من البيانات G أن: }
$$

$$
\text { إذ أن p هي رتبة البيان G. }
$$

2. بعض خواص المسافة العرضية

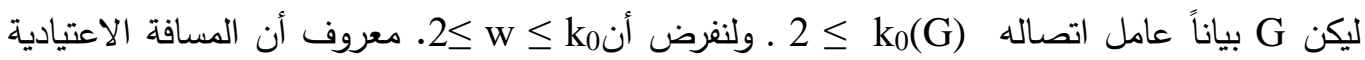

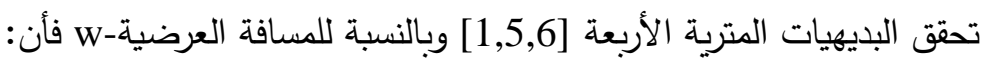

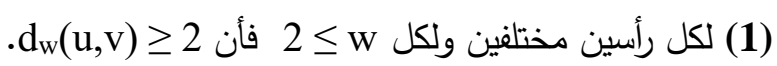

$$
\mathrm{d}_{\mathrm{w}}(\mathrm{u}, \mathrm{v})=\mathrm{d}_{\mathrm{w}}(\mathrm{v}, \mathrm{u})(\mathbf{2})
$$

(3) بالنسبة لأقل مسافة عرضية-w نعرف:

$\mathrm{m}_{\mathrm{w}}(\mathrm{G})=\min _{\mathrm{u}, \mathrm{v} \in \mathrm{V}(\mathrm{G})} \mathrm{d}_{\mathrm{w}}(\mathrm{u}, \mathrm{v})$

واضح أن mo أذا وفقط أذا كان خصر البيان G 2 هو 3 أو 4.

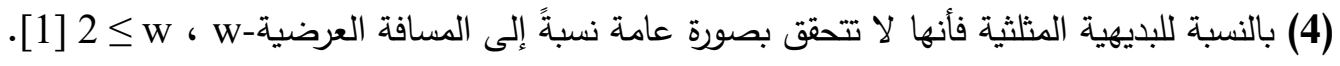


(5) معلوم أنه بالنسبة للمسافة الاعتيادية فأن قطر البيان G يكون مساويا إلى الواحد أذا وفقط أذا كان G بياناً تاما

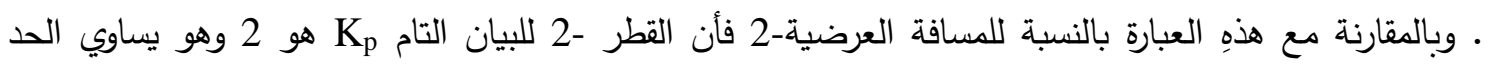

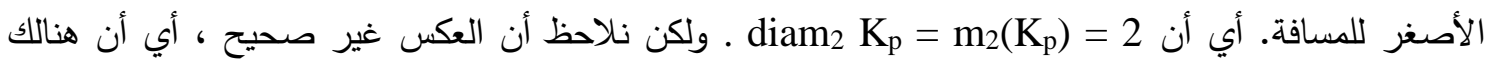

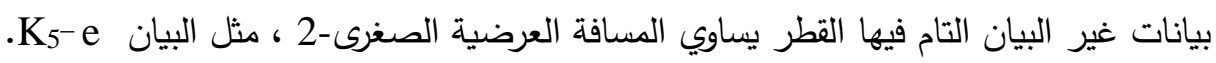

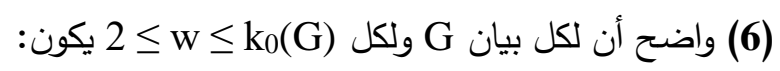

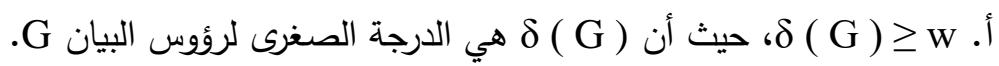

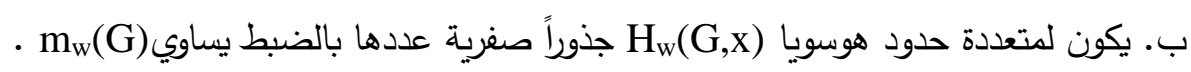

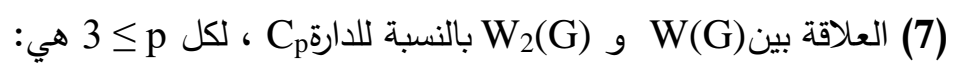
$\mathrm{W}_{2}\left(\mathrm{C}_{\mathrm{p}}\right)+\mathrm{W}\left(\mathrm{C}_{\mathrm{p}}\right)=\frac{1}{2} \mathrm{P}^{2}(\mathrm{P}-1)$

$\mathrm{W}\left(\mathrm{C}_{\mathrm{p}}\right)=\frac{1}{8} \mathrm{P}\left(\mathrm{P}^{2}-1\right), \mathrm{W}_{2}\left(\mathrm{C}_{\mathrm{p}}\right)=\frac{1}{8} \mathrm{P}(\mathrm{P}-1)(3 \mathrm{P}-1)$

وذلك لأنه: إذا كان P عدداً فردياً فأن [3,7 ]

$\mathrm{W}_{2}\left(\mathrm{C}_{\mathrm{p}}\right)=\frac{1}{8} \mathrm{P}^{2}(3 \mathrm{P}-4), \mathrm{W}\left(\mathrm{C}_{\mathrm{p}}\right)=\frac{1}{8} \mathrm{P}^{3}$

وبالجمع نحصل على النتيجة المطلوبة.

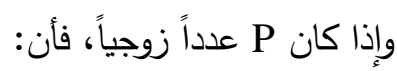

$\mathrm{W}_{2}\left(\mathrm{C}_{\mathrm{p}}\right)+\mathrm{W}\left(\mathrm{C}_{\mathrm{p}}\right)=\frac{1}{2} \mathrm{P}^{2}(\mathrm{P}-1)$

إذاً

وبالمثل فأن لدينا العبارة الآتية لكل بيان G بعامل اتصال لا يقل عن 2 : عبارة 2.1 : إذا كان G بياناً برتبة p وله عامل اتصال لا يقل عن 2 فأن: $\mathrm{W}_{2}(\mathrm{G})+\mathrm{W}(\mathrm{G}) \leq \frac{1}{2} \mathrm{P}(\mathrm{P}-1) . \mathrm{c}(\mathrm{G})$,

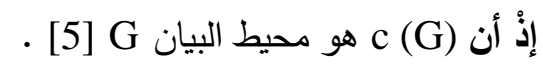

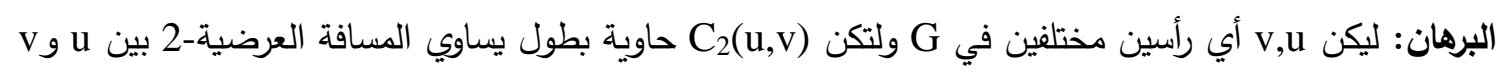
$\mathrm{d}_{2}(\mathrm{u}, \mathrm{v})=\ell\left(\mathrm{C}_{2}(\mathrm{u}, \mathrm{v})\right)$.

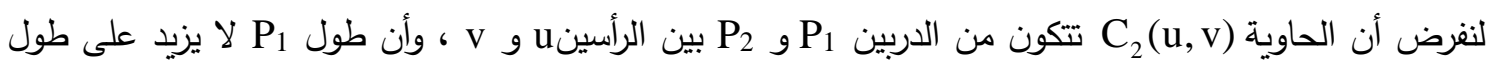
$\ell\left(\mathrm{P}_{1}\right) \leq \ell\left(\mathrm{P}_{2}\right)$ P P2 (u, $=\ell\left(\mathrm{P}_{2}\right)$ $\mathrm{d}_{2}(\mathrm{u}, \mathrm{v})=\ell\left(\mathrm{P}_{2}\right)$ $\mathrm{d}_{2}(\mathrm{u}, \mathrm{v}) \leq \mathrm{c}(\mathrm{G})-\ell\left(\mathrm{P}_{1}\right)$ ولما كان C(P واضتح أن

$\mathrm{d}(\mathrm{u}, \mathrm{v}) \leq \ell\left(\mathrm{P}_{2}\right)$ وذالك بموجب تعريف المسافة الاعتيادية بين الرأسين. لذلك فأن: $\mathrm{d}_{2}(\mathrm{u}, \mathrm{v}) \leq \mathrm{c}(\mathrm{G})-\mathrm{d}(\mathrm{u}, \mathrm{v})$ 
$\mathrm{d}_{2}(\mathrm{u}, \mathrm{v})+\mathrm{d}(\mathrm{u}, \mathrm{v}) \leq \mathrm{c}(\mathrm{G})$,

لكل رأسين مختلفين و v في G. وبأخذ المجموع لكل أزواج الرؤوس غير المرتبة في G ، نحصل على:

$\sum_{u, v \in V(G)} d_{2}(u, v)+\sum_{u, v \in V(G)} d(u, v) \leq \frac{1}{2} P(P-1) \cdot c(G)$,

$\mathrm{W}_{2}(\mathrm{G})+\mathrm{W}(\mathrm{G}) \leq \frac{1}{2} \mathrm{P}(\mathrm{P}-1) \cdot \mathrm{c}(\mathrm{G})$.

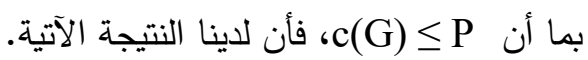
نتيجة 2.2 : كل بيان G بعامل اتصال لا يقل عن2 وبرتبة P يكون

$\mathrm{W}_{2}(\mathrm{G})+\mathrm{W}(\mathrm{G}) \leq \frac{1}{2} \mathrm{P}^{2}(\mathrm{P}-1)$

مسألة. لكل بيان G برتبة P وعامل اتصال لا يقل عن 2، فأن: $\mathrm{W}_{2}(\mathrm{G}) \leq \mathrm{W}_{2}\left(\mathrm{C}_{\mathrm{p}}\right) \quad\left[=\frac{1}{8} \mathrm{P}^{2}(3 \mathrm{P}-4)\right] . ?$

$\mathrm{W}(\mathrm{G}) \leq \mathrm{W}\left(\mathrm{P}_{\mathrm{p}}\right)$,

معروف بالنسبة للمسافة الاعتيادية أن لكل بيان G برتبة P يكون.

وأن المساواة تتحقق فقط عندما يكون G = P (الدرب برتبة P) ولقد وضعت المسألة في أعلاه مقارنةً بذالك، ولكنها تحتاج إلى برهان، وتركت لدأل لدراسة مستقبلية.

3. متعددة حدود هوسويا-2 لبيان سلسلة خماسية الحلقات خطية

ليكن G بيان سلسة خماسية الحلقات خطية مكونة من m من الحلقات كما هو موضح في الشكل 3.1.

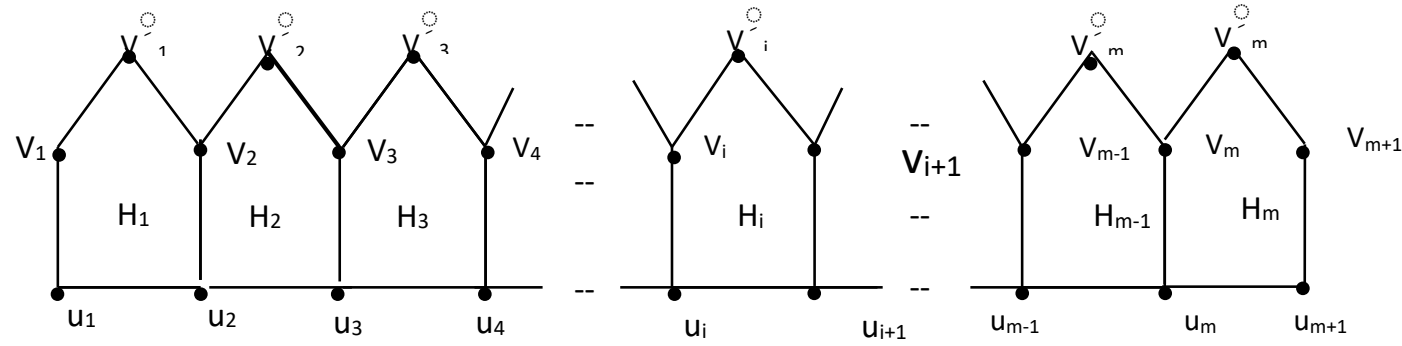

Gm

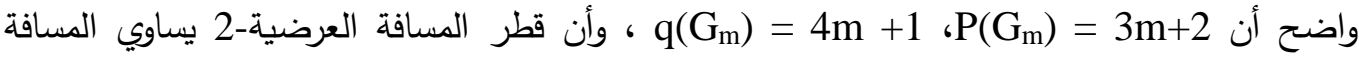

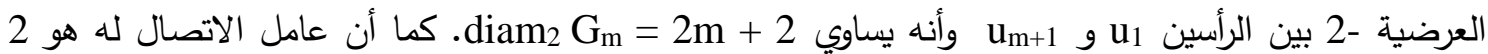

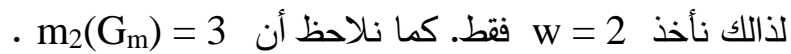
سنحاول أيجاد متعددة حدود هوسويا -2 للبيان G ساستعمال الاستقراء الرياضي على عدد الحلقات

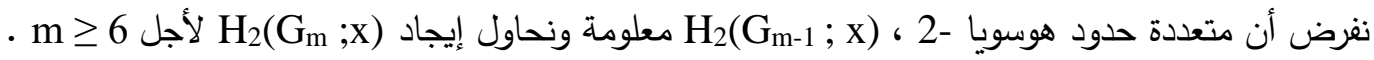
$\mathrm{H}_{2}\left(\mathrm{G}_{\mathrm{m}} ; \mathrm{x}\right)=\mathrm{H}_{2}\left(\mathrm{G}_{\mathrm{m}-1} ; \mathrm{x}\right)+\mathrm{H}_{2}\left(\mathrm{C}_{5} ; \mathrm{x}\right)+\mathrm{F}_{\mathrm{m}}(\mathrm{x})-\mathrm{x}^{4}$, بملاحظة G و G G

$\mathrm{F}_{\mathrm{m}}(\mathrm{x})=\sum_{\mathrm{u}, \mathrm{v}} \mathrm{x}^{\mathrm{d}_{2}(\mathrm{u}, \mathrm{v})}$, 
$\mathrm{v} \in \mathrm{V}\left(\mathrm{G}_{\mathrm{m}-1}\right)-\left\{\mathrm{v}_{\mathrm{m}}, \mathrm{u}_{\mathrm{m}}\right\}, \mathrm{u} \in \mathrm{A}=\left\{\mathrm{v}_{\mathrm{m}}, \mathrm{v}_{\mathrm{m}+1}, \mathrm{u}_{\mathrm{m}+1}\right\}$.

وأن المجموع يؤخذ على كل رأسين u و v vيث أن

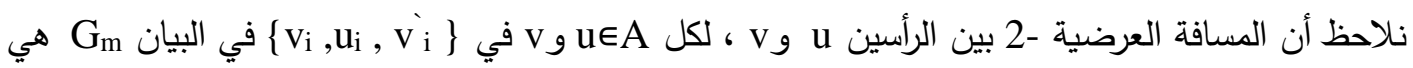
نفسها في الدارة بين الرأس vim وكل من رؤوس المجموعة $\mathrm{d}_{2}\left(\mathrm{v}_{\mathrm{m}}^{\prime}, \mathrm{u}_{\mathrm{i}}\right)=2+2(\mathrm{~m}-\mathrm{i}), \quad 1 \leq \mathrm{i} \leq \mathrm{m}-1$ ل

$$
\begin{aligned}
& \mathrm{d}_{2}\left(\mathrm{v}_{\mathrm{m}}^{\prime}, \mathrm{v}_{\mathrm{i}}\right)=\left\{\begin{array}{c}
1+2(\mathrm{~m}-\mathrm{i}), \quad 1 \leq \mathrm{i} \leq \mathrm{m}-4, \mathrm{f} \\
4+(\mathrm{m}-\mathrm{i}), \mathrm{i}=\mathrm{m}-3, \mathrm{~m}-2, \mathrm{~m}-1, \mathrm{~d}, \mathrm{y}
\end{array}\right\} \\
& \mathrm{d}_{2}\left(\mathrm{v}_{\mathrm{m}}^{\prime}, \mathrm{v}_{\mathrm{i}}\right)=\left\{\begin{array}{c}
2(\mathrm{~m}-\mathrm{i}) \\
5+(\mathrm{m}-\mathrm{i}), \mathrm{i}=\mathrm{m}-4, \mathrm{~m}-3, \mathrm{~m}-2, \mathrm{~m}-1, \mathrm{~d}, \mathrm{~d}
\end{array}\right\}
\end{aligned}
$$

ثم نجد المسافة العرضية -2 بين الرأس Vm+1 وكل من رؤوس A

$\mathrm{d}_{2}\left(\mathrm{v}_{\mathrm{m}+1}, \mathrm{u}_{\mathrm{i}}\right)=3+2(\mathrm{~m}-\mathrm{i}) \quad$ ألجل

$\mathrm{d}_{2}\left(\mathrm{v}_{\mathrm{m}+1}, \mathrm{v}_{\mathrm{i}}\right)=2+2(\mathrm{~m}-\mathrm{i}), \quad$ لأجل1

$\mathrm{d}_{2}\left(\mathrm{v}_{\mathrm{m}+1}, \mathrm{v}_{\mathrm{i}}^{\prime}\right)=\left\{\begin{array}{l}1+2(\mathrm{~m}-\mathrm{i}), 1 \leq \mathrm{i} \leq \mathrm{m}-3, \mathrm{f} \\ 4+(\mathrm{m}-\mathrm{i}), \mathrm{i}=\mathrm{m}-2, \mathrm{~m}-1 . \mathrm{l}\end{array}\right\}$

وأخيراً نجد المسافة العرضية -2 بين الرأس um+1 وكل من رؤوس A A فنحصل على:

$\mathrm{d}_{2}\left(\mathrm{u}_{\mathrm{m}+1}, \mathrm{u}_{\mathrm{i}}\right)=4+2(\mathrm{~m}-\mathrm{i}), 1 \leq \mathrm{i} \leq \mathrm{m}-1$ لأجل

$\mathrm{d}_{2}\left(\mathrm{u}_{\mathrm{m}+1}, \mathrm{v}_{\mathrm{i}}\right)=3+2(\mathrm{~m}-\mathrm{i}), \quad$ أجل لأن $1 \leq \mathrm{i} \leq \mathrm{m}-1$

$\mathrm{d}_{2}\left(\mathrm{u}_{\mathrm{m}+1}, \hat{\mathrm{v}}_{1}\right)=2+2(\mathrm{~m}-\mathrm{i}), 1 \leq \mathrm{i} \leq \mathrm{m}-1$ لأجل

$F_{m}(x)=\sum_{i=1}^{m-1}\left(3 x^{2+2(m-i)}+2 x^{3+2(m-i)}+x^{4+2(m-i)}\right)$

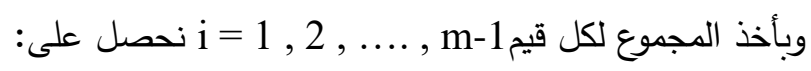

$$
\begin{aligned}
& +\sum_{i=1}^{m-3} x^{1+2(m-i)}+\sum_{i=1}^{m-4} x^{1+2(m-i)} \\
& +\sum_{i=1}^{m-5} x^{2(m-i)}+\left(x^{6}+x^{5}\right)+\left(x^{7}+x^{6}+x^{5}\right)+\left(x^{9}+x^{8}+x^{7}+x^{6}\right) .
\end{aligned}
$$

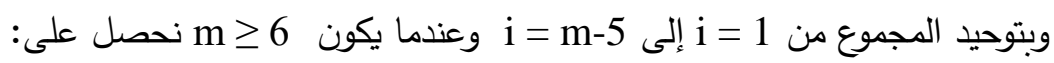

$F_{m}(x)=3 x^{4}+4 x^{5}+7 x^{6}+5 x^{7}+5 x^{8}+5 x^{9}+4 x^{10}+2 x^{11}+x^{12}$

$$
+\sum_{i=1}^{m-5} x^{2(m-i)}\left(3 x^{2}+2 x^{3}+x^{4}+2 x+1\right), \quad m \geq 6
$$

$\mathrm{F}_{\mathrm{m}}(\mathrm{x})=\mathrm{A}+\left(\mathrm{x}^{4}+2 \mathrm{x}^{3}+3 \mathrm{x}^{2}+2 \mathrm{x}+1\right) \sum_{\mathrm{i}=1}^{\mathrm{m}-5} \mathrm{x}^{2(\mathrm{~m}-\mathrm{i})}, \quad \mathrm{m} \geq 6$ 


$$
\begin{aligned}
& A=3 x^{4}+4 x^{5}+7 x^{6}+5 x^{7}+5 x^{8}+5 x^{9}+4 x^{10}+2 x^{11}+x^{12} \\
& \text { حيث أن: } \\
& \text { وبتكرار تطبيق العملية هذه على G-1 } \\
& \mathrm{H}_{2}\left(\mathrm{G}_{\mathrm{m}} ; \mathrm{x}\right)=\mathrm{H}_{2}\left(\mathrm{G}_{\mathrm{m}-2} ; \mathrm{x}\right)+2 \mathrm{H}_{2}\left(\mathrm{C}_{5} ; \mathrm{x}\right)+\mathrm{F}_{\mathrm{m}-1}(\mathrm{x})+\mathrm{F}_{\mathrm{m}}(\mathrm{x})-2 \mathrm{x}^{4}, \mathrm{~m} \geq 7 \\
& \text { وهكذا نستمر بتكرار العملية حتى نحصل على: } \\
& \mathrm{H}_{2}\left(\mathrm{G}_{\mathrm{m}} ; \mathrm{x}\right)=\mathrm{H}_{2}\left(\mathrm{G}_{5} ; \mathrm{x}\right)+(\mathrm{m}-5) \mathrm{H}_{2}\left(\mathrm{C}_{5} ; \mathrm{x}\right)-(\mathrm{m}-5) \mathrm{x}^{4}+\sum_{\mathrm{k}=6}^{\mathrm{m}} \mathrm{F}_{\mathrm{k}}(\mathrm{x}) \text {. }
\end{aligned}
$$

$$
\begin{aligned}
& F_{k}(x)=A+\left(x^{4}+2 x^{3}+3 x^{2}+2 x+1\right) \sum_{i=1}^{k-5} x^{2(k-i)}, k \geq 6 \\
& \sum_{\mathrm{k}=6}^{\mathrm{m}} \mathrm{F}_{\mathrm{k}}(\mathrm{x})=(\mathrm{m}-5) \mathrm{A}+\left(\mathrm{x}^{4}+2 \mathrm{x}^{3}+3 \mathrm{x}^{2}+2 \mathrm{x}+1\right) \sum_{\mathrm{k}=6}^{\mathrm{m}} \sum_{\mathrm{i}=1}^{\mathrm{k}-5} \mathrm{x}^{2(\mathrm{k}-\mathrm{i})}, \\
& \text { إذا كان m=m فأن هذا المجموع يكون صفراً. } \\
& \text { مما تقدم نحصل على المبرهنة الآتية: } \\
& \text { مبرهنة3.1. إذا كان m > } 6 \text { فأن: } \\
& \mathrm{H}_{2}\left(\mathrm{G}_{\mathrm{m}} ; \mathrm{x}\right)=\mathrm{H}_{2}\left(\mathrm{G}_{5} ; \mathrm{x}\right)+(\mathrm{m}-5) \mathrm{H}_{2}\left(\mathrm{C}_{5} ; \mathrm{x}\right)-(\mathrm{m}-5) \mathrm{x}^{4}+(\mathrm{m}-5) \mathrm{A} \\
& +\left(\mathrm{x}^{4}+2 \mathrm{x}^{3}+3 \mathrm{x}^{2}+2 \mathrm{x}+1\right) \sum_{\mathrm{k}=6}^{\mathrm{m}} \sum_{\mathrm{i}=1}^{\mathrm{k}-5} \mathrm{x}^{2(\mathrm{k}-\mathrm{i})} \text {, }
\end{aligned}
$$

إذ أن:

$A=x^{4}\left(3+4 x+7 x^{2}+5 x^{3}+5 x^{4}+5 x^{5}+4 x^{6}+2 x^{7}+x^{8}\right)$.

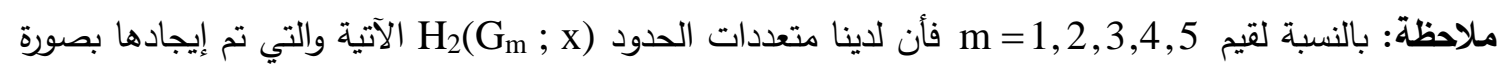

$$
\begin{aligned}
\mathrm{H}_{2}\left(\mathrm{G}_{1} ; \mathrm{x}\right)= & \mathrm{H}_{2}\left(\mathrm{C}_{5} ; \mathrm{x}\right)=5 \mathrm{x}^{3}+5 \mathrm{x}^{4} \\
\mathrm{H}_{2}\left(\mathrm{G}_{2} ; \mathrm{x}\right)= & 2 \mathrm{x}^{3}\left(5+6 \mathrm{x}+2 \mathrm{x}^{2}+\mathrm{x}^{3}\right) \\
\mathrm{H}_{2}\left(\mathrm{G}_{3} ; \mathrm{x}\right)= & 15 \mathrm{x}^{3}+19 \mathrm{x}^{4}+8 \mathrm{x}^{5}+9 \mathrm{x}^{6}+3 \mathrm{x}^{7}+\mathrm{x}^{8} \\
\mathrm{H}_{2}\left(\mathrm{G}_{4} ; \mathrm{x}\right)= & 20 \mathrm{x}^{3}+26 \mathrm{x}^{4}+12 \mathrm{x}^{5}+16 \mathrm{x}^{6}+8 \mathrm{x}^{7}+6 \mathrm{x}^{8}+2 \mathrm{x}^{9}+\mathrm{x}^{10} \\
\mathrm{H}_{2}\left(\mathrm{G}_{5} ; \mathrm{x}\right)= & 25 \mathrm{x}^{3}+33 \mathrm{x}^{4}+16 \mathrm{x}^{5}+23 \mathrm{x}^{6}+13 \mathrm{x}^{7}+11 \mathrm{x}^{8}+7 \mathrm{x}^{9}+5 \mathrm{x}^{10}+2 \mathrm{x}^{11} \\
& +\mathrm{x}^{12}
\end{aligned}
$$

مباشرة: - مبرة نتيجة 3.2. إذا كان m m 6 فان:

$$
\begin{aligned}
\mathrm{H}_{2}\left(\mathrm{G}_{\mathrm{m}} ; \mathrm{x}\right) & =\left(\mathrm{x}^{4}+2 \mathrm{x}^{3}+3 \mathrm{x}^{2}+2 \mathrm{x}+1\right) \sum_{\mathrm{k}=6}^{\mathrm{m}} \sum_{\mathrm{i}=1}^{\mathrm{k}-5} \mathrm{x}^{2(\mathrm{k}-\mathrm{i})}+\left[5 \mathrm{mx} \mathrm{x}^{3}+(7 \mathrm{~m}-2) \mathrm{x}^{4}+(4 \mathrm{~m}-4) \mathrm{x}^{5}\right. \\
& +(7 \mathrm{~m}-12) \mathrm{x}^{6}+(5 \mathrm{~m}-12) \mathrm{x}^{7}+(5 \mathrm{~m}-14) \mathrm{x}^{8}+(5 \mathrm{~m}-18) \mathrm{x}^{9}+(4 \mathrm{~m}-15) \mathrm{x}^{10} \\
& \left.+(2 \mathrm{~m}-8) \mathrm{x}^{11}+(\mathrm{m}-4) \mathrm{x}^{12}\right] .
\end{aligned}
$$




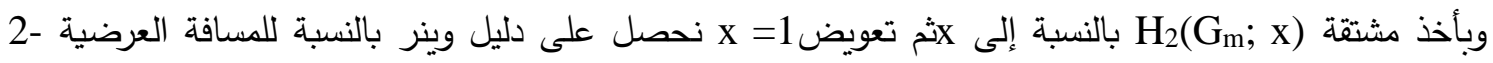
للبيان Gو، وكما هو مبين في النتيجة الآتية : $W_{2}\left(G_{m}\right)=3 m^{3}+9 m^{2}+35 m-24$ نتيجة 3.3. إذا كان m 6 ف فان :

$$
\begin{aligned}
\left(4 \mathrm{x}^{3}+6 \mathrm{x}^{2}+\right. & 6 \mathrm{x}+2) \sum_{\mathrm{k}=6}^{\mathrm{m}} \sum_{\mathrm{i}=1}^{\mathrm{k}-5} \mathrm{x}^{2(\mathrm{k}-\mathrm{i})} \\
& +\left(\mathrm{x}^{4}+2 \mathrm{x}^{3}+3 \mathrm{x}^{2}+2 \mathrm{x}+1\right) \sum_{\mathrm{k}=6}^{\mathrm{m}} \sum_{\mathrm{i}=1}^{\mathrm{k}-5} 2(\mathrm{k}-\mathrm{i}) \mathrm{x}^{2 \mathrm{k}-2 \mathrm{i}-1} \\
& +\left[15 \mathrm{mx}^{2}+4(7 \mathrm{~m}-2) \mathrm{x}^{3}+5(4 \mathrm{~m}-4) \mathrm{x}^{4}+6(7 \mathrm{~m}-12) \mathrm{x}^{5}\right. \\
& +7(5 \mathrm{~m}-12) \mathrm{x}^{6}+8(5 \mathrm{~m}-14) \mathrm{x}^{7}+9(5 \mathrm{~m}-18) \mathrm{x}^{8} \\
& \left.+10(4 \mathrm{~m}-15) \mathrm{x}^{9}+11(2 \mathrm{~m}-8) \mathrm{x}^{10}+12(\mathrm{~m}-4) \mathrm{x}^{11}\right] .
\end{aligned}
$$

البرهان: باشتقاق (3.3) بالنسبة إلى x نحصل على:

$$
\begin{aligned}
& \text { وبالتعويض عن x بواحد نحصل على دليل وينر -2 للبيان Gm وكما يأتي: } \\
& \mathrm{W}_{2}\left(\mathrm{G}_{\mathrm{m}}\right)=18 \sum_{\mathrm{k}=6}^{\mathrm{m}} \sum_{\mathrm{i}=1}^{\mathrm{k}-5}(1)+9 \sum_{\mathrm{k}=6}^{\mathrm{m}} \sum_{\mathrm{i}=1}^{\mathrm{k}-5} 2(\mathrm{k}-\mathrm{i})+[299 \mathrm{~m}-744] \\
& =299 \mathrm{~m}-744+18 \sum_{\mathrm{k}=6}^{\mathrm{m}}(\mathrm{k}-5)+9 \sum_{\mathrm{k}=6}^{\mathrm{m}}\left[2 \sum_{\mathrm{i}=1}^{\mathrm{k}-5} \mathrm{k}-2 \sum_{\mathrm{i}=1}^{\mathrm{k}-5} \mathrm{i}\right] \\
& =299 \mathrm{~m}-744+18 \sum_{\mathrm{k}=6}^{\mathrm{m}} \mathrm{k}-90(\mathrm{~m}-5)+9 \sum_{\mathrm{k}=6}^{\mathrm{m}}\left[2 \mathrm{k}(\mathrm{k}-5)-2 \cdot \frac{1}{2}(\mathrm{k}-4)(\mathrm{k}-5)\right] \\
& =299 \mathrm{~m}-744+18 \cdot \frac{1}{2}(\mathrm{~m}+6)(\mathrm{m}-5)-90(\mathrm{~m}-5)+9 \sum_{\mathrm{k}=6}^{\mathrm{m}}\left(\mathrm{k}^{2}-\mathrm{k}-20\right) \\
& =9 \mathrm{~m}^{2}+218 \mathrm{~m}-564+9 \sum_{\mathrm{k}=6}^{\mathrm{m}} \mathrm{k}^{2}-9 \sum_{\mathrm{k}=6}^{\mathrm{m}} \mathrm{k}-180(\mathrm{~m}-5) \\
& =9 \mathrm{~m}^{2}+38 \mathrm{~m}+336-9 \cdot \frac{1}{2}(\mathrm{~m}+6)(\mathrm{m}-5)+9 \sum_{\mathrm{k}=6}^{\mathrm{m}} \mathrm{k}^{2} \text {. } \\
& =9 m^{2}+38 m+336-\frac{9}{2}\left(m^{2}+m-30\right)+9\left[\frac{1}{6} m(m+1)(2 m+1)-55\right] \\
& =\frac{1}{6}\left(2 m^{3}+3 m^{2}+m\right)-55 \text {. }
\end{aligned}
$$

من المفيد إيجاد معدل المسافة العرضية ـ 2 للبيان Gm . نتيجة 3.4 لكل m 7 فان: $\mu_{2}\left(G_{m}\right) \leq \frac{2}{3} m+2.02$

البرهان: بما أن عدد رؤوس Gm 2 +3m فانه من النتيجة 3.3 نحصل على

$$
\begin{aligned}
\mu_{2}\left(G_{m}\right) & =W_{2}\left(G_{m}\right) /\left(\begin{array}{c}
3 m+2 \\
2
\end{array}\right) \\
& =2\left(3 m^{3}+9 m^{2}+35 m-24\right) /(3 m+2)(3 m+1)
\end{aligned}
$$




$$
=\frac{6 m^{3}+18 m^{2}+70 m-48}{9 m^{2}+9 m+2}
$$

$\mu_{2}\left(G_{m}\right)=\frac{2}{3} m+\frac{4}{3}+\frac{69}{100}-c, \quad 7 \leq m \quad l$

وبالقسمة، نحصل على

$\mu_{2}\left(G_{m}\right) \leq \frac{2}{3} m+2.02$

إذ أن c كمية موجبة، لذلك فان:

4. متعددات حدود هوسويا ـ 2 لسلسلة خماسية الحلقات متناوبة خطية:

ليكن G من الحلقات الخماسية والتي تثكل سلسلة خطية فيها الحلقات متتاوبة البية

بالنسبة للرأس الثنائي الدرجة كما هو مبين في الثكلين 4.1 ، 4.2 ـ
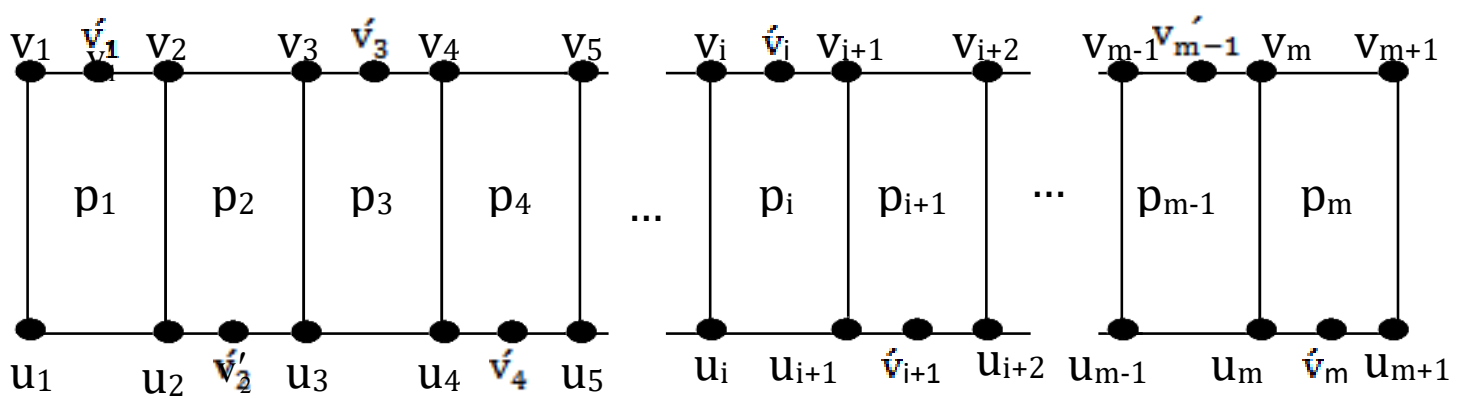

الثكل 4.1 بيان سلسلة خماسية الحلقات متتاوبة خطية G

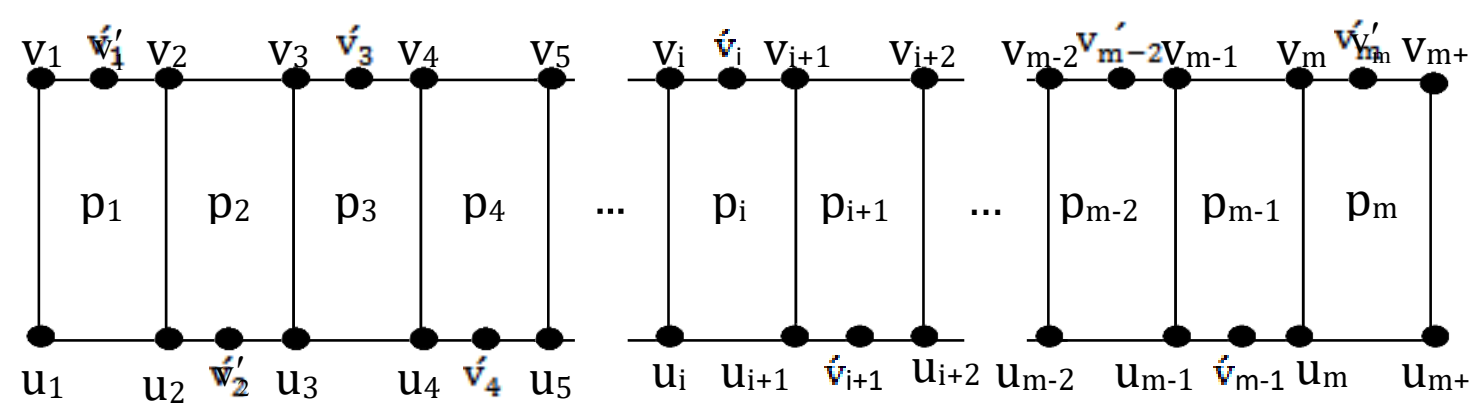

الثكل 4.2 بيان سلسلة خماسية الحلقات متتاوبة خطية G فيث أن m فردي

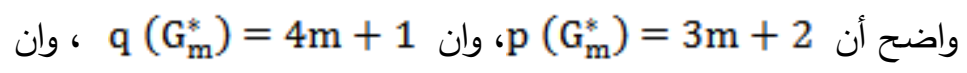

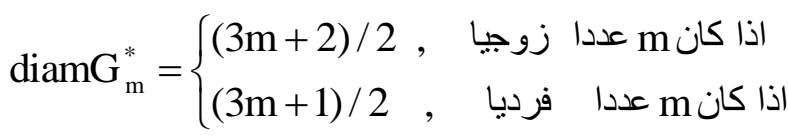

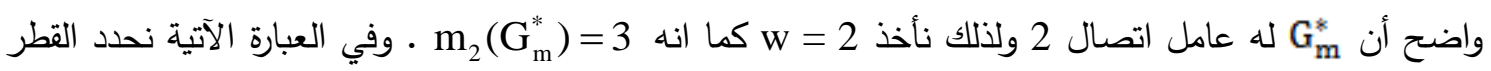
بالنسبة للمسافة العرضية ـ 2 ـ 2. العبارة 4.1. لكل Gان فان القطر بالنسبة للمسافة العرضية . 2هو

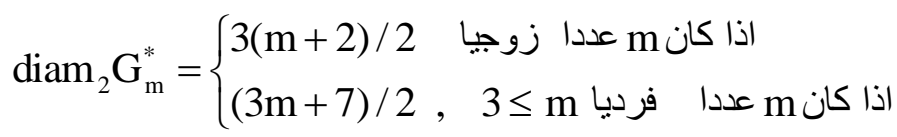




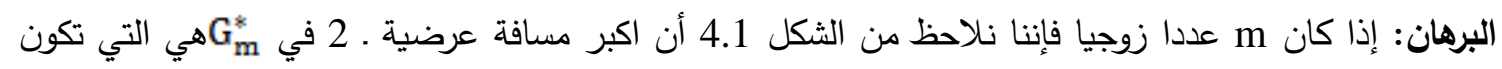

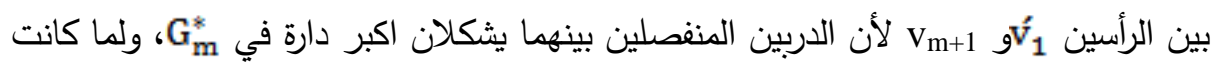
$\mathrm{d}_{2}\left(\dot{v}_{1}, \mathrm{v}_{\mathrm{m}+1}\right)=3+\mathrm{m}+\frac{\mathrm{m}}{2}=3(\mathrm{~m}+2) / 2$.

$\operatorname{diam}_{2} \mathrm{G}_{\mathrm{m}}^{*}=\frac{3}{2}(\mathrm{~m}+2)$

لذلك فانه عندما يكون m زوجيا فان

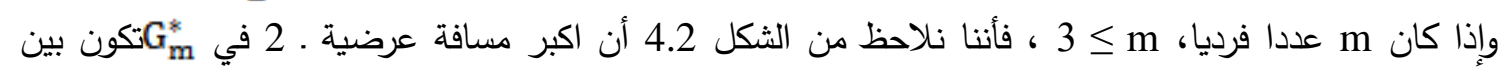
الرأسين $\mathrm{d}_{2}\left(\dot{\mathrm{v}}_{1}, \mathrm{v}_{\mathrm{m}}^{\prime}\right)=4+\mathrm{m}+\frac{\mathrm{m}-1}{2}=(3 \mathrm{~m}+7) / 2_{,}$ جميع رؤوس البيان G ولمان لذلك فانه عندما يكون m فرديا فان: $\operatorname{diam}_{2} \mathrm{G}_{\mathrm{m}}^{*}=(3 \mathrm{~m}+7) / 2$.

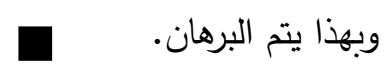
لأجل إيجاد صيغة لمتعددة حدود هوسايا ـ 2 للبيان Gوف نحتاج إلى المأخوذة الآتية.

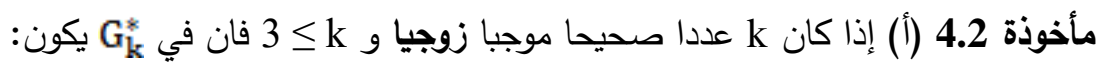
$F_{k}(x)=\sum_{u \in A, v \in A_{k}} x^{d_{2}(u, v)}=x^{\frac{3}{2} k+1}\left(2 x^{2}+4 x+3\right)$.

حيث أن

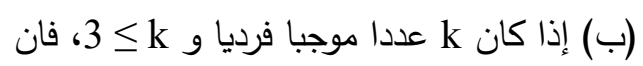

$$
\mathrm{F}_{\mathrm{k}}(\mathrm{x})=\mathrm{x}^{3(\mathrm{k}+1) / 2}\left(\mathrm{x}^{2}+3 \mathrm{x}+5\right) .
$$

البرهان: (أ) في Gk نحسب المسافة العرضية . 2 بين كل رأس في A وكل رأس في A وهذه مبينة في الجدول

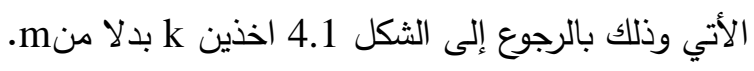

\begin{tabular}{|l|l|l|l|l|l|l|}
\hline$(\mathrm{u}, \mathrm{v})$ & $\mathrm{d}_{2}(\mathrm{u}, \mathrm{v})$ & $(\mathrm{u}, \mathrm{v})$ & $\mathrm{d}_{2}(\mathrm{u}, \mathrm{v})$ & $(\mathrm{u}, \mathrm{v})$ & $\mathrm{d}_{2}(\mathrm{u}, \mathrm{v})$ \\
\hline$\left(\mathrm{v}_{1}, \mathrm{v}_{\mathrm{k}+1}\right)$ & $2+\frac{3}{2} \mathrm{k}$ & $\left(\mathrm{u}_{1}, \mathrm{v}_{\mathrm{k}+1}\right)$ & $1+\frac{3}{2} \mathrm{k}$ & $\left(\mathrm{v}_{1}^{\prime}, \mathrm{v}_{\mathrm{k}+1}\right)$ & $3+\frac{3}{2} \mathrm{k}$ \\
$\left(\mathrm{v}_{1}, \mathrm{u}_{\mathrm{k}+1}\right)$ & $1+\frac{3}{2} \mathrm{k}$ & $\left(\mathrm{u}_{1}, \mathrm{u}_{\mathrm{k}+1}\right)$ & $2+\frac{3}{2} \mathrm{k}$ & $\left(\mathrm{v}_{1}, \mathrm{u}_{\mathrm{k}+1}\right)$ & $2+\frac{3}{2} \mathrm{k}$ \\
$\left(\mathrm{v}_{1}, \hat{\mathrm{v}}_{\mathrm{k}}^{\prime}\right)$ & $2+\frac{3}{2} \mathrm{k}$ & $\left(\mathrm{u}_{1}, \hat{\mathrm{v}}_{\mathrm{k}}^{\prime}\right)$ & $3+\frac{3}{2} \mathrm{k}$ & $\left(\hat{\mathrm{v}}_{1}, \hat{\mathrm{v}}_{\mathrm{k}}\right)$ & $1+\frac{3}{2} \mathrm{k}$ \\
\hline
\end{tabular}

من هذا الجدول نحصل على Fk (x) كما هي معطاة في (4.1) .

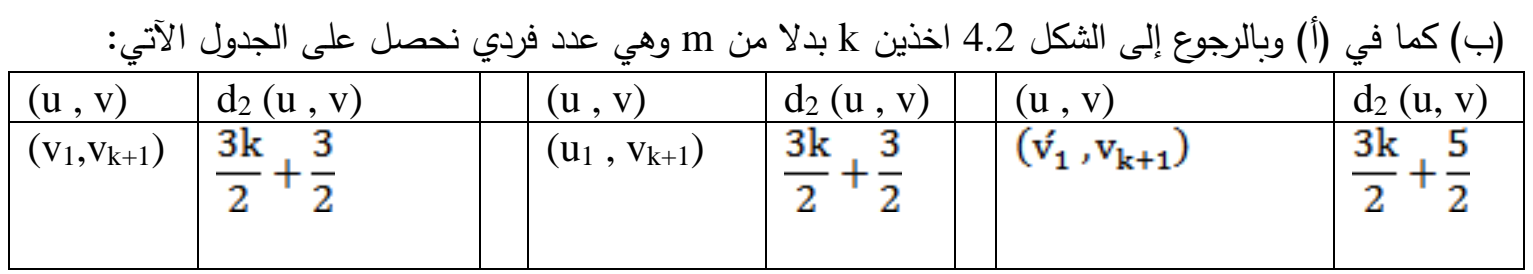




\begin{tabular}{|l|l|l|l|l|l|l|}
\hline$\left(\mathrm{v}_{1}, \mathrm{u}_{\mathrm{k}+1}\right)$ & $\frac{3 \mathrm{k}}{2}+\frac{3}{2}$ & & $\left(\mathrm{u}_{1}, \mathrm{u}_{\mathrm{k}+1}\right)$ & $\frac{3 \mathrm{k}}{2}+\frac{5}{2}$ & $\left(\mathrm{v}_{1}, \mathrm{u}_{\mathrm{k}+1}\right)$ & $\frac{3 \mathrm{k}}{2}+\frac{3}{2}$ \\
$\left(\mathrm{v}_{1}, \hat{\mathrm{v}}_{\mathrm{k}}^{\prime}\right)$ & $\frac{3 \mathrm{k}}{2}+\frac{5}{2}$ & $\left(\mathrm{u}_{1}, \hat{\mathrm{v}}_{\mathrm{k}}^{\prime}\right)$ & $\frac{3 \mathrm{k}}{2}+\frac{3}{2}$ & $\left(\hat{\mathrm{v}}_{1}, \hat{\mathrm{v}}_{\mathrm{k}}\right)$ & $\frac{3 \mathrm{k}}{2}+\frac{7}{2}$ \\
\hline
\end{tabular}

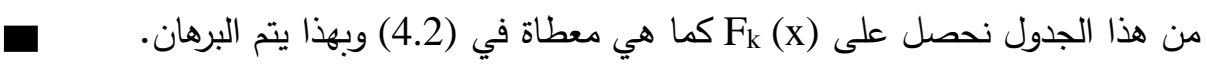
المبرهنة 4.3 لكل m 3 3 فان متعددة حدود هوسايا -2 هي:

$$
\begin{aligned}
\mathrm{H}_{2}\left(\mathrm{G}_{\mathrm{m}}^{*} ; \mathrm{x}\right) & =(\mathrm{m}-1)\left(2 \mathrm{x}^{6}+4 \mathrm{x}^{5}+7 \mathrm{x}^{4}+5 \mathrm{x}^{3}\right)+5 \mathrm{x}^{4}+5 \mathrm{x}^{3}+\sum_{\mathrm{j}=2}^{\left[\frac{\mathrm{m}}{2}\right]}\left[(\mathrm{m}+1-2 \mathrm{j})\left(2 \mathrm{x}^{3}+4 \mathrm{x}^{2}+3 \mathrm{x}\right)\right. \\
& \left.+(\mathrm{m}+2-2 \mathrm{j})\left(\mathrm{x}^{2}+3 \mathrm{x}+5\right)\right] \mathrm{x}^{3 \mathrm{j}}
\end{aligned}
$$

البرهان: أولا نجد علاقة تكرارية لـ من الثكل 4.1 (أو 4.2) نلاحظ أن البيانات $\mathrm{U}_{\mathrm{m}}^{*}-\left\{\mathrm{v}_{\mathrm{m}}^{\prime}, \mathrm{v}_{\mathrm{m}+1}, \mathrm{u}_{\mathrm{m}+1}\right\}, \mathrm{G}_{\mathrm{m}}^{*}-\left\{\mathrm{v}_{1}^{\prime}, \mathrm{v}_{1}, \mathrm{u}_{1}\right\}$ و $\mathrm{G}_{\mathrm{m}-1}^{*}$ $\mathrm{H}_{2}\left(\mathrm{G}_{\mathrm{m}}^{*} ; \mathrm{x}\right)=2 \mathrm{H}_{2}\left(\mathrm{G}_{\mathrm{m}-1}^{*} ; \mathrm{x}\right)-\mathrm{H}_{2}\left(\mathrm{G}_{\mathrm{m}-2}^{*}\right)+\mathrm{F}_{\mathrm{m}}(\mathrm{x})$ وان كلا منها يحتوي على حيث أن Fm معطاة في المأخوذة 4.2 وحسب كون m زوجي أو فردي.

$$
\mathrm{H}_{2}\left(\mathrm{G}_{\mathrm{m}-1}^{*} ; \mathrm{x}\right)=2 \mathrm{H}_{2}\left(\mathrm{G}_{\mathrm{m}-2}^{*} ; \mathrm{x}\right)-\mathrm{H}_{2}\left(\mathrm{G}_{\mathrm{m}-3}^{*} ; \mathrm{x}\right)+\mathrm{F}_{\mathrm{m}-1}(\mathrm{x})
$$

$$
\begin{aligned}
& \mathrm{H}_{2}\left(\mathrm{G}_{\mathrm{m}}^{*} ; \mathrm{x}\right)=3 \mathrm{H}_{2}\left(\mathrm{G}_{\mathrm{m}-2}^{*} ; \mathrm{x}\right)-2 \mathrm{H}_{2}\left(\mathrm{G}_{\mathrm{m}-3}^{*} ; \mathrm{x}\right)+2 \mathrm{~F}_{\mathrm{m}-1}(\mathrm{x})+\mathrm{F}_{\mathrm{m}}(\mathrm{x}) \\
& =3\left[2 \mathrm{H}_{2}\left(\mathrm{G}_{\mathrm{m}-3}^{*} ; \mathrm{x}\right)-\mathrm{H}_{2}\left(\mathrm{G}_{\mathrm{m}-4}^{*} ; \mathrm{x}\right)+\mathrm{F}_{\mathrm{m}-2}(\mathrm{x})\right]-2 \mathrm{H}_{2}\left(\mathrm{G}_{\mathrm{m}-3}^{*} ; \mathrm{x}\right)+2 \mathrm{~F}_{\mathrm{m}-1}(\mathrm{x})+\mathrm{F}_{\mathrm{m}}(\mathrm{x}) \\
& =4 \mathrm{H}_{2}\left(\mathrm{G}_{\mathrm{m}-3}^{*} ; \mathrm{x}\right)-3 \mathrm{H}_{2}\left(\mathrm{G}_{\mathrm{m}-4}^{*} ; \mathrm{x}\right)+3 \mathrm{~F}_{\mathrm{m}-2}(\mathrm{x})+2 \mathrm{~F}_{\mathrm{m}-1}(\mathrm{x})+\mathrm{F}_{\mathrm{m}}(\mathrm{x}) \text {. } \\
& \text { وهكذا بعد j من الخطوات نتوصل إلى: } \\
& \mathrm{H}_{2}\left(\mathrm{G}_{\mathrm{m}}^{*} ; \mathrm{x}\right)=(\mathrm{j}+1) \mathrm{H}_{2}\left(\mathrm{G}_{\mathrm{m}-\mathrm{j}}^{*} ; \mathrm{x}\right)-\mathrm{jH}_{2}\left(\mathrm{G}_{\mathrm{m}-\mathrm{j}-1}^{*} ; \mathrm{x}\right)+\sum_{\mathrm{i}=0}^{\mathrm{j}-1}(\mathrm{i}+1) \mathrm{F}_{\mathrm{m}-\mathrm{i}}(\mathrm{x}) \\
& \text { وأخيرا بعد } 2 \text { - من الخطوات، أي نعوض } 2 \text { - m = m في العلاقة السابقة نحصل على: } \\
& \mathrm{H}_{2}\left(\mathrm{G}_{\mathrm{m}}^{*} ; \mathrm{x}\right)=(\mathrm{m}-1) \mathrm{H}_{2}\left(\mathrm{G}_{2}^{*} ; \mathrm{x}\right)-(\mathrm{m}-2) \mathrm{H}_{2}\left(\mathrm{G}_{1}^{*} ; \mathrm{x}\right)+\sum_{\mathrm{i}=0}^{\mathrm{m}-3}\left[(\mathrm{i}+1) \mathrm{F}_{\mathrm{m}-\mathrm{i}}(\mathrm{x})\right. \text {. }
\end{aligned}
$$

كما يمكن أن نبسط المجموع بتعويض k= m - i فحصل على:

$\sum_{\mathrm{i}=0}^{\mathrm{m}-3}(\mathrm{i}+1) \mathrm{F}_{\mathrm{m}-\mathrm{i}}(\mathrm{x})=\sum_{\mathrm{k}=\mathrm{m}}^{3}(\mathrm{~m}+1-\mathrm{k}) \mathrm{F}_{\mathrm{k}}(\mathrm{x})=\sum_{\mathrm{k}=3}^{\mathrm{m}}(\mathrm{m}+1-\mathrm{k}) \mathrm{F}_{\mathrm{k}}(\mathrm{x})$.

$\mathrm{k}=3,4,5,6, \ldots, \mathrm{m}-2, \mathrm{~m}-1, \mathrm{~m}$. 


$$
\begin{aligned}
& \mathrm{k}=\left\{\begin{array}{lll}
4,6,8, & \ldots & , \mathrm{m}-2, \mathrm{~m} \\
3,5,7, & \ldots & , \mathrm{m}-1
\end{array}\right. \\
& \text { عندما يكون m عددا زوجيا، أما عندما يكون m ع أسدا فرديا فأن التجزئة لقيم كالأتي: } \\
& \mathrm{k}=\left\{\begin{array}{c}
4.6,8, \ldots, \mathrm{m}-1 \\
3,5,7, \ldots, \mathrm{m}
\end{array}\right.
\end{aligned}
$$

وهكذا عندما يكون m زوجيا نحصل من المأخوذة 4.2 على:

$$
\begin{aligned}
\sum_{k=3}^{m}(m+1-k) F_{k}(x)= & \sum_{j=2}^{\frac{m}{2}}(m+1-2 j)\left(2 x^{3}+4 x^{2}+3\right) x^{3 j+1} \\
& +\sum_{j=2}^{\frac{m}{2}}(m+2-2 j)\left(x^{2}+3 x+5\right) x^{3 j}
\end{aligned}
$$

وهذه هي كما في الصيغة (4.3) عندما يكون m عددا زوجيا. وعندما يكون m عددا فرديا نستخدم المأخوذة 4.2 فنحصل على على

$$
\begin{aligned}
\sum_{k=3}^{m}(m+1-k) F_{k}(x)= & \sum_{j=2}^{\frac{m-1}{2}}(m+1-2 j)\left(2 x^{3}+4 x^{2}+3\right) x^{3 j+1} \\
& +\sum_{j=2}^{\frac{m+1}{2}}(m+2-2 j)\left(x^{2}+3 x+5\right) x^{3 j} .
\end{aligned}
$$

لاحظ انه عندما نعوض j m

صفراً، ولذلك يمكننا جعل الحد الأعلى للمجموع الأول في الصيغة (4.3) عندما يكون m عددا فرديا. وبالرجوع إلى (4.4) واستخدام (4.5) نفسها. وبهذا يتم البرهان. النتيجة الآتية تعطيب دليل وينر . 2 للبيان

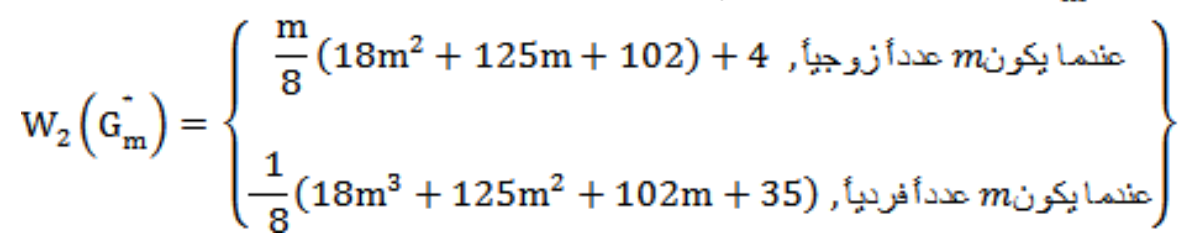

البرهان : باستخدام المبرهنة 4.3 واشتقاق متعددة الحدود $\mathrm{H}_{2}\left(\mathrm{G}_{\mathrm{m}}{ }^{\mathrm{F}} ; \mathrm{x}\right.$ بالنسبة إلى X ثم تعويض x=1 نحصل

$$
\begin{aligned}
\mathrm{W}_{2}\left(\mathrm{G}_{\mathrm{m}}^{\mathrm{F}}\right)=(\mathrm{m}-1)(75)+35 & \\
& +\sum_{\mathrm{j}=2}^{\left[\frac{\mathrm{m}}{2}\right]}\{[(\mathrm{m}+1-2 \mathrm{j})(17)+(\mathrm{m}+2-2 \mathrm{j})(5)] \\
& +3 \mathrm{j}[(\mathrm{m}+1-2 \mathrm{j})(9)+(\mathrm{m}+2-2 \mathrm{j})(9)]\}
\end{aligned}
$$




$$
\begin{aligned}
& =75 \mathrm{~m}-40+\sum_{\mathrm{j}=2}^{\left\lceil\frac{\mathrm{m}}{2}\right\rceil}\{22 \mathrm{~m}+27-44 \mathrm{j}+3 \mathrm{j}(18 \mathrm{~m}+27-36 \mathrm{j})\} \\
& =75 \mathrm{~m}-40+\sum_{\mathrm{j}=2}^{\left[\frac{\mathrm{m}}{2}\right]}\left\{22 \mathrm{~m}+27+(54 \mathrm{~m}+37) \mathrm{j}-108 \mathrm{j}^{2}\right\} \\
& \text { (1) إذا كان m عددا زوجياً فان } \\
& W_{2}\left(G_{m}^{*}\right)=75 m-40+\left(\frac{m}{2}-1\right)(22 m+27)+(54 m+37) \sum_{j=2}^{\frac{m}{2}} j-108 \sum_{j=2}^{\frac{m}{2}} j^{2} \\
& =\frac{1}{8}\left(18 \mathrm{~m}^{3}+125 \mathrm{~m}^{2}+102 \mathrm{~m}+32\right)=\frac{\mathrm{m}}{8}\left(18 \mathrm{~m}^{2}+125 \mathrm{~m}+102\right)+4 \text {. } \\
& \mathrm{W}_{2}\left(\mathrm{G}_{\mathrm{m}}^{\mathrm{F}}\right)=57 \mathrm{~m}-40+\left(\frac{\mathrm{m}-1}{\underset{\frac{\mathrm{m}+1}{2}}{2}}\right)(22 \mathrm{~m}+27)-(54 \mathrm{~m}+37)+108 \\
& +(54 m+37) \sum_{j=1}^{\frac{m+1}{2}} j-108 \sum_{j=1}^{\frac{m+1}{2}} j^{2} \\
& =11 \mathrm{~m}^{2}+\frac{47}{2} \mathrm{~m}+\frac{35}{2}+\frac{1}{8}\left(\mathrm{~m}^{2}+4 \mathrm{~m}+3\right)(18 \mathrm{~m}-35) \\
& =\frac{1}{8}\left(18 \mathrm{~m}^{3}+125 \mathrm{~m}^{2}+102 \mathrm{~m}+35\right) \text {. }
\end{aligned}
$$




$$
\begin{aligned}
& \text { المصادر } \\
& \text { الياس، شوان سلو عبدال (2010)، "حول متعددات هوسويا للمسـافة العرضية في البيانات "، رسالة } \\
& \text { ماجستير (غير منشورة) الجامعة الحرة - فرع نينوى. } \\
& \text { علي، علي عزيز (1983)، مقدمـة في نظريـة البيـان، ط 1 1، الموصل: مؤسسـة دار الكتب بجامعـة } \\
& \text { الموصل. } \\
& \text { البيانات الخاصة"، مجلة الرافدين لعلوم الحاسبات والرياضيات، } 4 \text { (2)، } 103 \text { - } 124 . \\
& \text { عزيز ، أسماء صلاح (2007)، " المسافة العرضية ومتعددات حدود وينر-w للبيان"، رسالة ماجستير ، } \\
& \text { (غير منشورة)، جامعة الموصل. }
\end{aligned}
$$

[5] Buckley, F. and Harary, F. (1990), Distance in Graphs, 1 st ed., New York, Addison- Wesley .

[6] Chartrand, G. and Lesniak, L. (1986), Graphs and Digraphs, 1 st ed., California, Wadsworth Inc.

[7] Gutman, I.(1993), "Some properties of the Wiener Polynomials", Graph Theory Notes of New York, XXV, 13-18 .

[8] Hosoya, H.(1988), "On some counting polynomials in Chemistry", Discrete Applied Math., 19, 239-257.

[9] Sagan, B.E., Yeh, Y.N., and Zhang, P. (1996), "The Wiener polynomial of a graph", Intr. J. Quantum. Chem., 60( 5), 959-969 . 